Ralph Savelsberg, Doctor, Faculty of Military Sciences, Netherlands

Defence Academy

\title{
AN ANALYSIS OF NORTH KOREA'S SATELLITE LAUNCHES
}

\begin{abstract}
In December of 2012, North Korea successfully launched a satellite, after failures in 1998, 2009 and 2012. The carrier missiles used during the last three launches are of a new design. Computer simulations of different models for these missiles, using information on the satellite trajectories, show that they require more advanced technology than North Korea has demonstrated previously. This knowledge allows an estimate of the performance of two road mobile ballistic missiles shown during parades in 2010 and 2012. The first, known as the Musudan, theoretically has a range that is considerably larger than the currently operational North Korean missiles. The second, the $K N-08$, however, is too heavy to be based on the rocket engines used in the missiles used for the satellite launches, which means that it is either a mockup or that North Korea is developing more powerful engines. The lack of a flight test program makes it unlikely that either of these missiles is close to being operational.
\end{abstract}

\section{Keywords}

Ballistic missiles, North Korea, computer simulations, missile defence

\section{Introduction}

On the 12th of December 2012 North Korea caused an international incident by successfully launching a satellite into orbit, in violation of UN Security Council resolutions (BBC, 2012). The satellite, known as the Kwangmyŏngsŏng 3-2, was launched into a polar orbit from a missile base in the northwest of the country, using a three-stage carrier missile known as the Unha-3-2 (see Fig. 1). This successful launch followed three earlier unsuccessful attempts to launch satellites. The first, in August 1998, used a three-stage missile. The ballistic missile that consists of the first two stages is known in the West as the Taepodong-1. During this attempt, the third stage of the missile failed (Postol, 2009). The second attempt, in April 2009, used a much larger three-stage missile known as the Unha-2. The satellite, named 
the Kwangmyŏngsŏng-2, was launched from the East-coast of North Korea in an easterly direction over Japan, towards the Pacific Ocean. It never reached its intended orbit, most likely also because the third stage of the missile failed to ignite (Covault, 2009). The successful December launch was a repeat of an attempted launch that took place on the 13th of April 2012, timed to celebrate Kim-Il-Sung's 100th birthday. The 100 kg Kwangmyŏngsŏng 3 satellite was to be launched into a circular polar orbit at an altitude of $500 \mathrm{~km}$. The Unha-3 carrier missile broke up in flight, due to an as yet unknown cause. There is conflicting information about the time at which the missile failed and about where the wreckage came down (Christy 2012a).

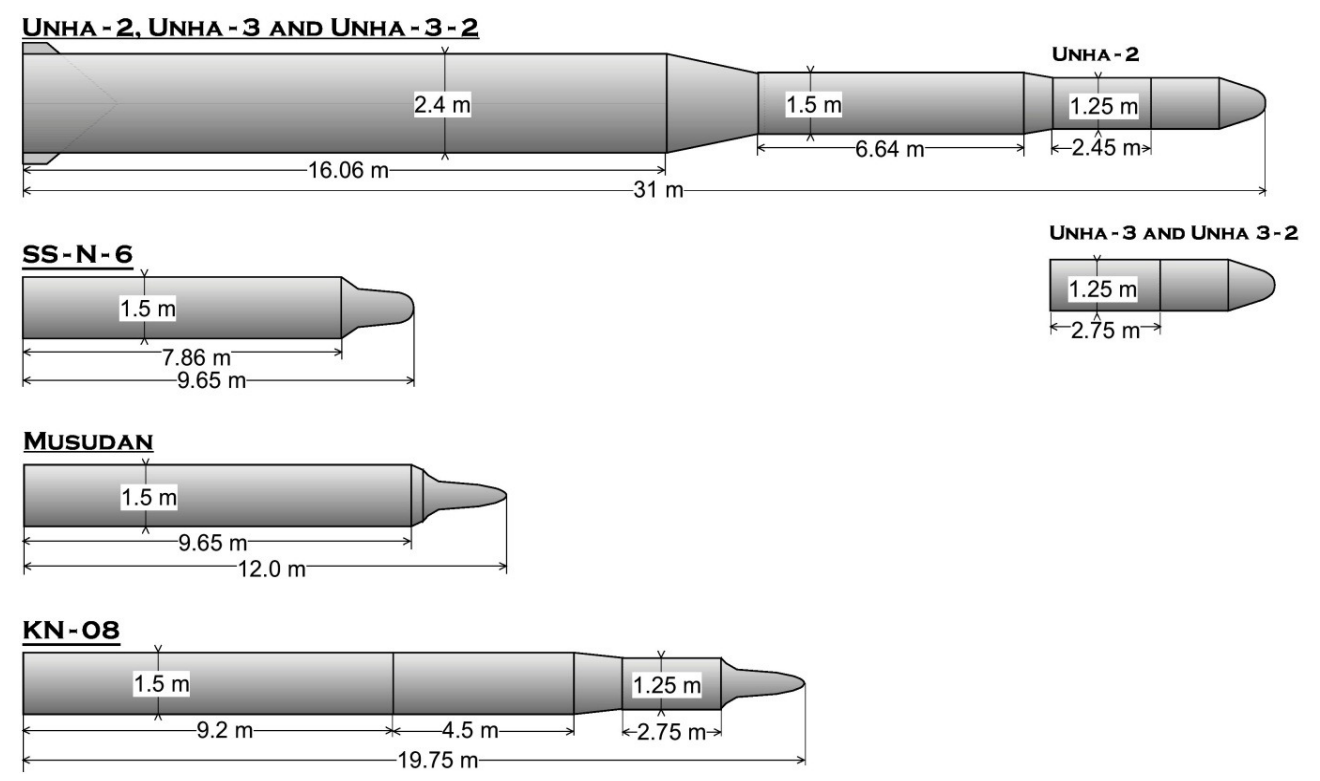

Figure 1: The Unha-2 and Unha-3/3-2 space launchers compared to the Soviet SS-N-6 medium-range ballistic missile and the North Korean Musudan and KN-08 missiles. The dimensions of the Unha-2 follow from Wright and Postol (2009), of the Unha-3 from Wright (2012) and of the SSN-6 from Pike (2011). The Musudan's dimensions follow from Lennox (2009) and the KN-08's dimensions from Hansen (2012).

Officially the satellites were intended for Earth-observation (KCNA, 2012), but North Korea's space program is widely seen as a cover for testing technology for intercontinental ballistic missiles (Pellerin, 2012). The first two stages of the Unha-2, Unha-3 and Unha-3-2 are associated with an 
intercontinental ballistic missile (ICBM) known as the Taepodong-2. This was unsuccessfully tested in July of 2006, exploding 40-42 s into its flight (Pinkerton, 2008). It does not seem likely that the North Koreans have mastered building miniaturized nuclear weapons, which are small and light enough to serve as the payload of their ballistic missiles. The country does have an active nuclear weapons program, however, and is suspected of collaborating with Iran in developing longer-range missiles (Hecker \& Carlin, 2012). The threat of North Korea developing an ICBM or exporting the technology to countries such as Iran is a major motivation behind the US and Europe developing a missile defence system (Sessions, 2008). The successful launch in December 2012 shows that North Korea is making progress, although the flight test program is still very limited and may essentially be a bluff (Schiller, 2012).

As part of an investigation of Iranian missile technology, Postol performed a detailed analysis of ballistic missiles developed by North Korea (Postol 2009). Most North Korean ballistic missiles are based on the technology of the Soviet Scud-B. North Korea has the ability to produce variants of the Scud and has developed and exported its own versions. The most advanced North Korean missile that is currently operational is the Nodong, which is also in service in Iran as the Shahab-3. Limitations of the technology mean that, if one wanted to build an ICBM with a sufficient range to reach the United States from North Korea or the United States from Iran, the resulting missile would end up being very large and heavy. Such a missile would be difficult to transport and launch using a mobile installation. During a parade in Pyongyang in October 2010, a new missile was shown, carried by a transporter erector launcher vehicle (TEL). The missile is variously known as the Musudan, BM-25, Taepodong-X or Nodong. ${ }^{1}$ At a first glance, it closely resembles the Soviet SS-N-6 (Pollack, 2010). The SS-N-6 is a liquid-fuelled submarine-launched ballistic missile that is no longer in service with the Russian military, but that is still considerably more advanced than the Scud. It has a lighter structure and more energetic fuel (Pike, 2011, Wright, 2010). In April 2012, shortly after the failed launch of the Unha-3, a second new road mobile missile was shown in a parade in the North Korean capital (Lewis, 2012a; Richardson, 2012a). It is known to Western analysts as the

\footnotetext{
${ }^{1}$ The North Korean designation of the missile is unknown, but in this article it will be referred to as the Musudan.
} 
$\mathrm{KN}-08$. It appears to be a longer multi-stage missile with a similar diameter as the Musudan. Both missiles are smaller than a hypothetical ICBM based on technology of the Scud and Nodong, but if these missiles were to use the more advanced technology of the SS-N-6, they would represent a larger threat than North Korea's current arsenal. The missile geometries are illustrated in Fig. 1.

Two parameters that indicate the technology level of the missiles, which are also used in the simulations, are the specific impulse and the fuel-structure ratio. For a liquid-fuelled missile, the propellant is a combination of a fuel and a separate oxidizer. The specific impulse is a measure of the amount of thrust that can be delivered per mass flow of the propellant.

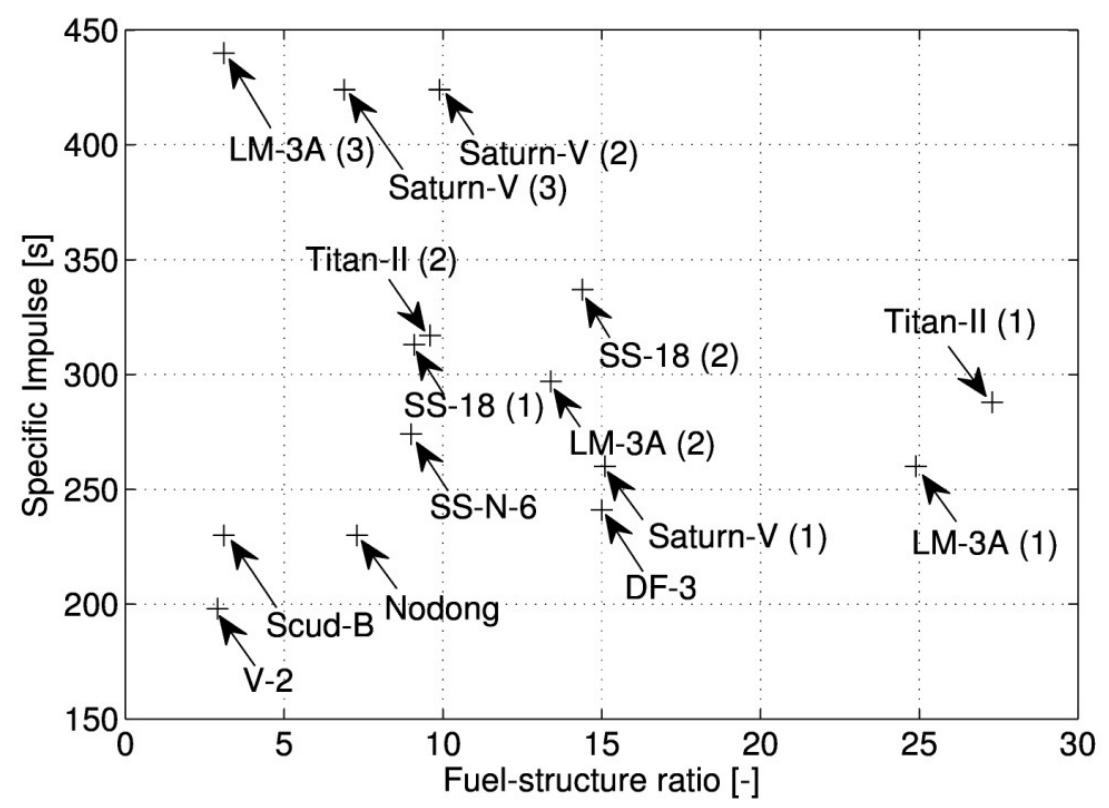

Figure 2: Data points show the specific impulse and the fuel-structure ratios for liquid-fuelled missiles: the Scud-B (Forden, 2007), Nodong (Vick, 2012) and SS-N-6 (Pike, 2011), as well as the US Saturn-V (NASA, 1968) and Chinese LM-3A (CGWIC, 2011) space launchers, the Chinese DF-3 medium-range ballistic missile, the US Titan-II ICBM and Soviet SS-18 ICBM (Fetter, 1990). For multi-staged missiles, the numbers between parentheses indicate the stage number. The world's first operational ballistic missile, the German WW-II V-2, is also shown (Fetter, 1990). 
It depends on the propellant, but is also affected by the engine nozzle design and the ambient pressure at the operating altitude of the missile, as well as, for instance, the presence of thrust-vectoring vanes in the nozzle. The fuelstructure ratio is the ratio of the propellant mass in a stage and the mass of the structure of the stage itself (excluding the payload). A more advanced missile will require less structural weight to carry a similar amount of propellant. This is illustrated in Fig. 2, which shows the specific impulse and fuelstructure ratios for a number of liquid-fuelled missiles. The figure clearly shows that these parameters for the Scud-B and Nodong are closer to the V-2 than to any of the other missiles. These missiles have a lower specific impulse and relatively low fuel-structure ratios. Only the fuel-structure ratios of the upper stages of the space launchers (LM-3A and Saturn-V) are lower than that of the Nodong, but these stages are designed to launch heavy payloads (of multiple tons). This requires a relatively heavy structure and their low structure factors are offset by their much larger specific impulse. The parameters for the SS-N-6 are much more in line with the more advanced missiles. There are indications that North Korea has access to the technology of the SS-N-6 (Postol, 2009; Lewis, 2012b), but there is disagreement on whether the Unha-2 and 3, the Musudan and the $\mathrm{KN}-08$ are indeed based on this technology (Schiller \& Schmucker, 2012a; Hansen, 2012). The intended trajectories of the Kwangmyŏngsŏng-2 and 3 satellites and the locations of the impact zones for the first two missile stages, announced by North Korea before the launch, are the key to answering the question whether or not North Korea indeed has access to the technology of the SS-N-6 or technology of a similar level.

In this paper the satellite orbits are compared to results of computer simulations of an Unha-2 missile model that is based either on the technology of the Scud or based on that of the SS-N-6. The computer model used for the simulations is similar to a model used by Forden (2007). Chapter 2 explains the differences and explains the parameters of the missiles that are required as inputs and how their values can be estimated from published values of similar missiles. In Chapter 3 the ranges of North Korea's existing ballistic missiles are presented and compared to the range of the SS-N-6, illustrating the performance gain that is possible with its more advanced technology and validating the computer simulations. In Chapter 4, simulations of different models for the Unha-2 are compared to the planned orbit of the 
Kwangmyŏngsŏng- 2 satellite. The results of the comparison are applied to the launches of the Unha-3 and Unha-3-2. The results of these simulations are used to shed light on the theoretical performance of the Musudan and $\mathrm{KN}-08$ ballistic missiles in Chapter 5 and the paper ends with a brief discussion of the results and conclusions.

\section{Simulating the missiles and their trajectories}

To simulate the missile trajectories, a computer program has been written in MATLAB/ Simulink that solves the equations of motion numerically. This involves calculating the forces that act on the missile as a function of time and calculating how the mass of the missile changes as fuel is consumed and missile stages are discarded. Forces included in the model are gravity, aerodynamic drag and thrust, illustrated in Fig. 3. The equations of motion, the coordinate system and the assumptions and simplifications used in the model are the same as those in an existing program by Forden (2007) and the reader is referred to his original paper for many of the details. This existing program, however, is not suitable for calculating the trajectories used to launch satellites.

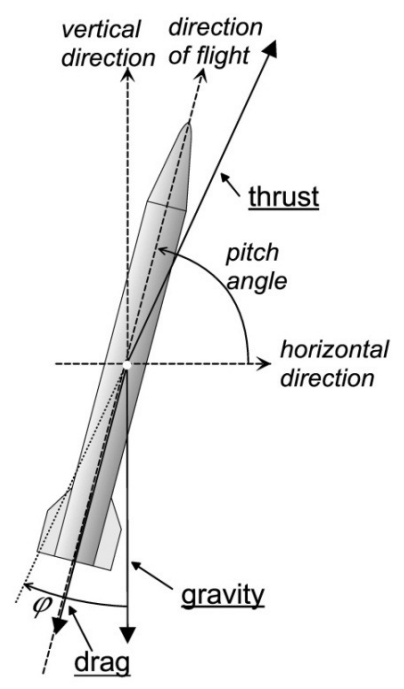

Figure 3: Forces on the missile modelled in the computer simulation. Drag is aligned with the missile axis, gravity points vertically down and the thrust, which is only present during the boost-phase, is offset from the vertical by an angle $\varphi$ that is a function of time. 
To calculate such trajectories, a number of changes and additions were made in the computer model, which will be explained in this chapter. A minor difference between the model used here and the program by Forden is that the latter uses an exponential atmosphere (with an exponential density profile), whereas the current simulations use the standard atmosphere instead (see Appendix A, Anderson, 2008).

The trajectory of a ballistic missile consists of three phases. The boost-phase is the time during which the rocket engines produce thrust. The missile continues its trajectory unpowered during the mid-course phase. For medium range ballistic missiles and ICBMs this phase will take place largely outside of the atmosphere. During the final phase, the so-called re-entry, the missile or its payload returns into the atmosphere. The impact point is mainly determined by the velocity, altitude and pitch angle (the angle between the trajectory and horizontal) at the end of the boost-phase. A larger range requires a larger velocity. For a maximum range trajectory, the pitch angle at the end of the boost-phase is approximately $45^{\circ}$ for relatively short range ballistic missiles and decreases for longer range missiles (Zarchan 1997). During the boost-phase, the pitch of a missile as a function of time -the socalled pitch-program - is determined by a balance between forces and moments. The thrust is angled away from the missile axis, which generates a moment. This moment results in an angle of attack between the missile axis and the direction of flight, causing aerodynamic lift and an associated aerodynamic force moment. The computer model does not take into account force moments and the only aerodynamic force that is included is drag. In the model, steering the missile is done by changing the angle $\varphi$ between the thrust vector and the vertical direction in time, see Fig. 3. Initially, the thrust is pointed in the vertical direction, with $\varphi=0$. In Forden's program and in the calculations of the maximum range of ballistic missiles reported here, after a few seconds, the angle $\varphi$ is increased linearly with time until it reaches a maximum value. ${ }^{2}$ The velocity, pitch angle and altitude at the end of the boost-phase are determined by this maximum value and the time at which the angle $\varphi$ reaches it. In the simulations, the range is maximized by

\footnotetext{
${ }^{2}$ In the current simulations, 5 seconds was chosen. Keeping the missile vertical during the first seconds of its flight limits the time spent in the densest part of the atmosphere.
} 
calculating new trajectories for different values of these two parameters. The equations of motion are solved in Earth-fixed coordinates, and they include (optional) corrections to account for the rotation of the Earth. The effect of the rotation is that the maximum range of a ballistic missile launched towards the east is larger than that of a similar missile launched west.

A space launch requires a different trajectory during the boost-phase, which requires changes to the model. North Korea has employed a so-called direct orbit insertion, in which the missile has to fly horizontally at the end of the boost-phase, with a velocity that matches the orbital velocity at the altitude that has been reached. At this point the thrust should longer have a vertical component, which means that $\varphi$ has to be $90^{\circ}$. In the calculations of orbit injection, after the first few seconds of the flight, $\varphi$ as a function of time now is a third-order polynomial function

$$
\begin{array}{ll}
0<t<t_{k} & \rightarrow \varphi=0^{\circ} \\
t \geq t_{k} & \rightarrow \varphi=90^{\circ} \cdot\left(1-\left(\frac{t-t_{b}}{t_{k}-t_{b}}\right)^{2}+\left(t-t_{k}\right)\left(t-t_{b}\right)(A t-B)\right) .
\end{array}
$$

In this function $t$ is the time since launch, $t_{k}$ and $t_{b}$ are, respectively, the time at which the pitch-over manoeuvre starts and the boost-phase ends and $A$ and $B$ are parameters that determine the shape of the curve. The polynomial was chosen such that at $t=t_{k}, \varphi=0^{\circ}$ and at $t=t_{b}, \varphi=90^{\circ}$, regardless of the values of $A$ and $B$. Out of the possible trajectories, the program needs to select the one that delivers the satellite to the desired orbit. For a circular orbit, the velocity at the end of the boost-phase needs to equal the orbital velocity (see, for instance Vallado, 2007)

$$
V_{\text {orbit }}=\sqrt{\frac{G M}{h_{\text {orbit }}+R}} .
$$

In this equation, $h_{\text {orbit }}$ is the altitude of the orbit above the Earth surface, $R$ is the radius of the Earth $\left(6.3781 \times 10^{6} \mathrm{~m}\right), G$ is the gravitational constant $\left(6.67398 \times 10^{-11} \mathrm{~m}^{3} \mathrm{~kg}^{-1} \mathrm{~s}^{-2}\right)$ and $M$ is the mass of the Earth $\left(5.97219 \times 10^{24}\right.$ $\mathrm{kg}$ ). S.I. units are used for all quantities throughout this paper. For a circular orbit at an altitude of $500 \mathrm{~km}, V_{\text {orbit }}=7.6 \mathrm{~km} / \mathrm{s}$. This is considerably larger than velocities reached by ballistic missiles. If the velocity at the end of the boost-phase is smaller than the orbital velocity, the missile will follow a suborbital trajectory and will return to Earth. If the velocity is larger, it will reach 
an elliptical orbit. In the simulation, to check whether the missile has reached orbit, the magnitude of the velocity at the end of the boost-phase is compared to the orbital velocity given by Eq.2. However, this equation applies to a nonrotating coordinate system and a correction needs to be made to the eastward velocity component of the missile to account for the rotation of the Earthfixed coordinate system

$$
V_{\text {east }}^{\prime}=V_{\text {east }}+\left(h_{\text {orbit }}+R\right) \cdot \omega_{\text {Earth }} \cdot \cos \phi .
$$

In this equation $V_{\text {east }}^{\prime}$ and $V_{\text {east }}$ are the eastward component of the velocity at the end of the boost-phase in the non-rotating and Earth-fixed coordinate systems, respectively, $\omega_{E \text { Earth }}$ is the rotation of the Earth $(2 \pi /(24 \cdot 3600)$ $\mathrm{rad} / \mathrm{s})$ and $\phi$ is the latitude reached at the end of the boost-phase. The effect of the rotation is that launching a satellite in an easterly direction, as in North Korea's test in 2009, for instance, requires a smaller velocity from the missile than launching a satellite of the same mass south into a polar orbit, as in the tests of 2012. The program has two different modes for choosing the orbit. In the first mode, the altitude of the orbit, $h_{\text {orbit }}$, is not pre-set. The values of $A$ and $B$ are varied and the resulting trajectories are calculated until the trajectory is found for which the pitch angle at the end of the boost-phase indeed is zero and the velocity equals $V_{\text {orbit }}$ for the altitude reached. In the second mode, $A$ and $B$ are varied until the boost phase ends at a pre-set altitude, with pitch-angle equal to zero. In that case, however, the velocity may not match $V_{\text {orbit }}$. In both modes, the simulation also calculates the trajectories followed by the first and second stages after they have been discarded, assuming that they do not tumble and that their drag-coefficient is the same as that of the complete missile. ${ }^{3}$

The parameters for the missile models used in the simulations are estimated using data from open sources. The program requires the diameter of the base of each missile stage for the calculation of the drag, as in Forden (2007), the payload mass and, for every stage $i$, the structure mass $m_{s, i}$, i.e. the mass of the stage without propellant, the total propellant mass in the stage $m_{p, i}$, the specific impulse $I_{s p, i}$ and the burn time $\Delta t_{i}$. Assuming that the mass flow $\dot{m}_{i}$

\footnotetext{
${ }^{3}$ The drag of the discarded stages is likely larger than that of the complete missile, because of their much blunter front ends. If the stages tumble, drag is increased even further. This means that the simulation will likely overestimate how far they travel.
} 
through the engine of a stage is constant during its burn, the thrust $T_{i}$ is given by (see, for instance, Zarchan 1997)

$$
T_{i}=I_{s p, i} \dot{m}_{i} g,
$$

in which $g$ is the gravitational acceleration $\left(9.8 \mathrm{~ms}^{-2}\right)$ and with the mass flow given by

$$
\dot{m}_{i}=\frac{m_{p, i}}{\Delta t_{i}}
$$

The nozzle of a rocket engine, which generates thrust by accelerating the exhaust gasses, is optimised for a particular range of altitudes and in reality the thrust therefore depends on altitude. For a given engine design, the $I_{s p}$ at sea level will be a few percent less than the $I_{s p}$ in space. In the program $I_{s p}$ is fixed for a given stage, however. The total mass $m_{i}$ of a stage decreases linearly during its burn, as fuel and oxidizer are consumed

$$
m_{i}=m_{s, i}+m_{p . i}-\dot{m}_{i}\left(t-t_{0, i}\right)
$$

in which $t$ is the time since launch and $t_{0, i}$ is the time at which stage $i$ was ignited. The total mass of the missile at any given time $t$ can be found by adding the payload mass and the structure- and propellant masses of stages that have not yet been discarded to Eq. 6. The values for the missile parameters used in the simulations are estimated from published values of similar missiles, using three assumptions. The first is that the fuel structureratio can be kept constant for a missile of a given technology level. In terms of the parameters in Equations 4 and 5, the fuel-structure ratio of a stage is

$$
\mu_{i}=\frac{m_{i, p}}{m_{i, s}} .
$$

The second assumption is that the total take-off mass of a stage, $m_{s, i}+m_{p . i}$, is proportional to the length of the stage, provided that the diameter remains unchanged. Finally, for a given liquid-fuelled rocket engine, the mass flow and $I_{s p}$ are assumed constant, which means that increasing the amount of propellant in a missile stage will not change the thrust, but instead will increase the burn time. For example, to estimate the values of the required parameters for a lengthened version of the Scud, the propellant mass, structure mass and the duration of the boost-phase for the basic Scud-B are multiplied by the ratio of the booster lengths. 
The numerical scheme used to solve the differential equations is a fixed timestep Bogacki-Shampine method, which is a standard numerical solver in MATLAB/Simulink (see Shampine 1997). To speed up computation times the default time-step is set to $1 \mathrm{~s}$. Using smaller time-steps or a higher order solver changes the maximum range or the altitude of the orbit less than $1 \%$.

\section{The North-Korean missile program}

Most of North Korean's ballistic missiles are derived from the Scud-B, most likely because developing new rocket engines, structures, materials and more energetic missile fuel is complicated and requires extensive tests. For the North-Korean developed Scud-C, the basic Scud design was extended; increasing range by increasing the fuel mass, but sacrificing payload (see Table 1).

Table 1: Data of North Korean missiles and the Soviet SS-N-6 for comparison. Data for the Scud-C were derived from data from the Scud-B, by keeping the total mass constant, but adding the payload mass saved to the booster mass while keeping the fuel-structure ratio constant.

\begin{tabular}{|l|r|r|r|r|r|} 
& & $\begin{array}{l}\text { Scud-B } \\
\text { (Forden, } \\
\text { 2007) }\end{array}$ & NK Scud-C & \multicolumn{1}{l|}{$\begin{array}{l}\text { Nodong } \\
\text { (Vick, 2007) }\end{array}$} & $\begin{array}{l}\text { SS-N-6 (Pike, } \\
\text { 2011) }\end{array}$ \\
\hline Input parameter & Symbol & & & & 650 \\
\hline Payload [kg] & & 1000 & 300 & 700 & 1.5 \\
\hline Diameter [m] & & 0.88 & 0.88 & 1.25 & 1350 \\
Empty mass [kg] & $m_{s, 1}$ & 1198 & 1393 & 1780 & 12200 \\
Propellant mass & $m_{p, 1}$ & 3699 & 4300 & 12913 & $274-290$ \\
[kg] & $I_{s p, 1}$ & 230 & 230 & 230 & 128.5 \\
Specific impulse [s] & $\Delta t_{1}$ & 75 & 87 & 110 & 9.0 \\
Burn time [s] & $\mu_{1}$ & 3.1 & 3.1 & 7.3 & \\
\hline Fuel-structure ratio & & & & & $2.34 \cdot 10^{3}$ \\
\hline Simulation result & & 2.93 & 528 & $1.30 \cdot 10^{3}$ & \\
\hline Range (without & & & & & \\
Earth rotation) & & & & \\
[km] & & & & & \\
\hline
\end{tabular}

This development did not require a new rocket engine. A different approach was used in the development of the Nodong. It is essentially an enlarged version of the Scud-B. Increasing the size increased the propellant mass and 
the structural mass, but, overall, the fuel-structure ratio was improved. The take-off weight of the Nodong exceeds the thrust of a Scud-engine and, to provide sufficient thrust, a scaled-up Scud engine was developed that can accommodate a larger mass flow. It is not clear whether the Nodong is an indigenous North Korean development of the Scud or whether its design was imported from the (former) Soviet-Union (Schiller 2012).

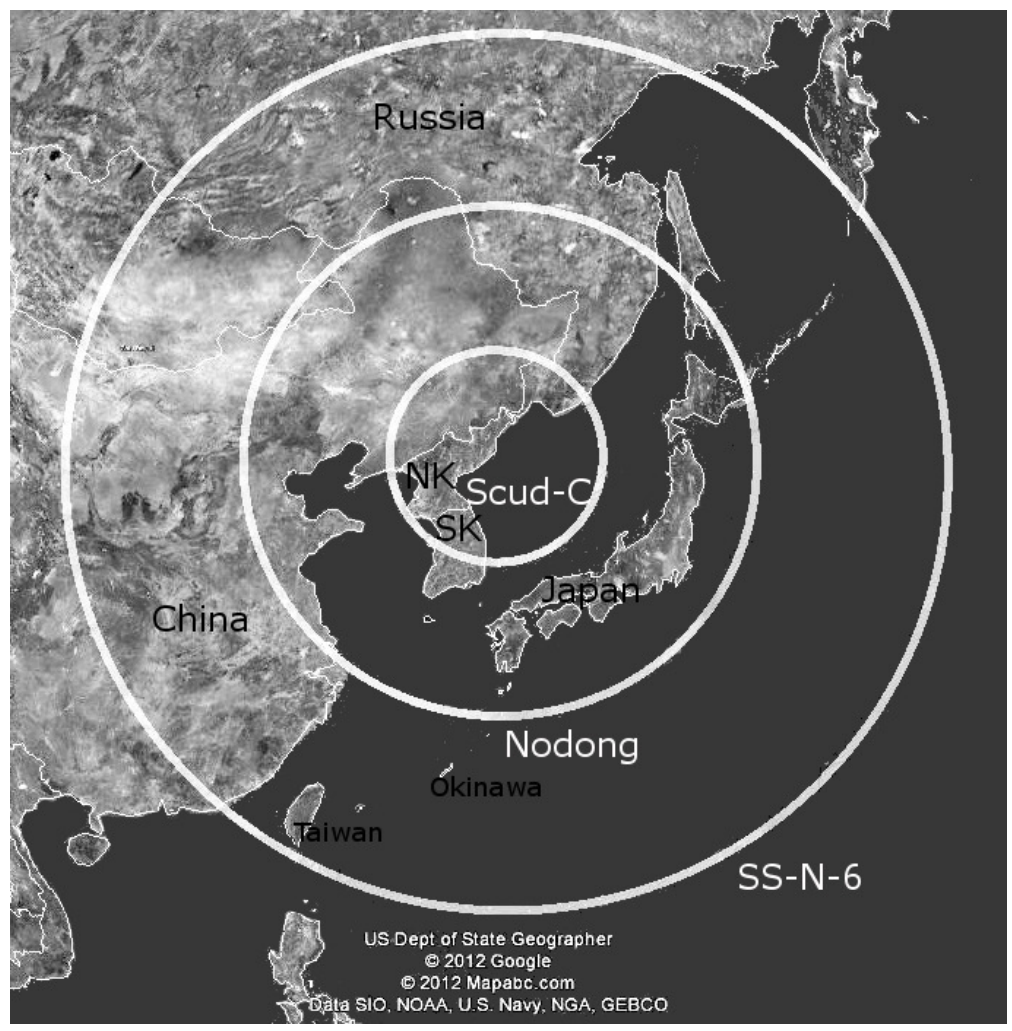

Figure 4: Missile ranges based on computer simulations. ${ }^{4}$ In the simulations, the missiles were launched from the Musudan-Ri missile site in the northeast of North Korea, but when launched from sites close to the border, the Scud-C can reach all of South Korea. The Nodong can reach most of Japan. A missile with the performance of the SS-N-6 can reach Okinawa and Taiwan. The visualisation was done using Google Earth.

To validate the results of the computer simulations and to illustrate how modifying the Scud-design has increased the performance of North Korea's missiles, maximum ranges have been calculated for the basic Scud-B, the

\footnotetext{
${ }^{4}$ At a first glance, the area of the Earth surface that the missile can reach from a given launch site is a circle, but it is slightly deformed due to the rotation of the Earth
} 
North Korean version of the Scud-C, and the Nodong. The data that are needed to calculate the ranges of these different ballistic missiles, their fuelstructure factors and the ranges that follow from the simulation are listed in Table 1. For comparison purposes, the range for the more advanced Soviet SS-N-6 was also calculated. The results are shown graphically in Fig. 4.

The increased range of the Scud-C allows it to reach all of South Korea from launch sites near the border, while the larger Nodong can reach most of Japan. An SS-N-6 would be able to reach Okinawa and Taiwan. Its range is almost twice the range of the Nodong, despite their similar take-off masses. This clearly shows the advantage of the higher $I_{s p}$ and the larger fuelstructure ratio, shown in Fig. 2. The calculated ranges are all within a few per cent of the published ranges of these missiles.

\section{The satellite launches}

In an attempt to convince the world that the satellite launch in April 2012 served peaceful purposes, Reporters were given unprecedented access to the base before the launch. Based on their photographs, the Unha-3 appears to be essentially the same missile as the Unha-2, except with a slightly longer third stage (Wright, 2012). Footage of the Unha-3-2 shows that, in turn, it is essentially a copy of the Unha-3. The satellite orbits were announced in advance. The Uhna-2 was to launch a satellite into a circular orbit towards the east at an altitude of $500 \mathrm{~km}$. The satellites launched with the Unha-3 and Unha-3-2 were to reach a circular polar orbit at $500 \mathrm{~km}$. Before the satellite launch in April 2009, North Korea published a NOTAM message, giving coordinates of the expected impact zones of the first and second stages (Global Security, 2009). Even though the launch failed, the second stage indeed did impact in the announced hazard zone (Covault, 2009). North Korea again announced hazard zones for the first and second stages of the Unha-3 (Williams, 2012) and the Unha-3-2 (Christy, 2012b). These hazard zones and the orbits offer crucial clues to the nature of the missile.

The question whether or not the Unha-2 is based on the SS-N-6 or the Scud/Nodong can be answered by calculating the trajectories for different missile models, based on the Nodong or the SS-N-6. Three different models of the Unha-2, listed in Table 2, are used in the simulations. For the most 
accurate missile model, the first and second stages end up in or closest to the hazard zones, and the satellite reaches a circular orbit at the desired altitude.

Table 2: Data for different models for the Unha-2 and the Unha-3 missile used in the simulations.

\begin{tabular}{|l|c|c|c|c|c|}
\hline Missile name & & Unha-2 & Unha-2 & Unha-2 & Unha-3 \\
\hline Model name & & U2a & U2b & U2c & U3 \\
\hline $2^{\text {nd }}$ stage & & SS-N-6 & Nodong & Nodong & SS-N-6 \\
\hline $3^{\text {rd }}$ stage & & SS-N-6 & SS-N-6 & Nodong & SS-N-6 \\
\hline Payload [kg] & & 300 & 300 & 300 & 300 \\
\hline Stage 1 & & & & & \\
\hline diameter [m] & $m_{s, 1}$ & 8649 & 2.4 & 2.4 & 2.4 \\
Empty mass [kg] & $m_{p, 1}$ & 65401 & 65401 & 8649 & 8649 \\
Propellant mass [kg] & $I_{s p, 1}$ & 220 & 220 & 220 & 65401 \\
Specific impulse [s] & $\Delta t_{1}$ & 118 & 118 & 118 & 118 \\
Burn time [s] & $\mu_{1}$ & 7.6 & 7.6 & 7.6 & 7.6 \\
\hline Fuel-structure ratio & & & & & \\
\hline stage 2 & & 1.5 & 1.5 & 1.5 & 1.5 \\
\hline Diameter [m] & $m_{s, 2}$ & 1655 & 1750 & 1750 & 1655 \\
Empty mass [kg] & $m_{p, 2}$ & 11895 & 12542 & 12542 & 11895 \\
Fuel mass [kg] & $I_{s p, 2}$ & 300 & 255 & 255 & 300 \\
Specific impulse [s] & $\Delta t_{2}$ & 122 & 110 & 110 & 122 \\
Burn time [s] & $\mu_{2}$ & 7.2 & 7.2 & 7.2 & 7.2 \\
\hline Fuel-structure ratio & & & & & \\
\hline stage 3 & & 1.25 & 1.25 & 1.25 & 1.40 \\
\hline Diameter [m] & $m_{s, 3}$ & 465 & 465 & 454 & 522 \\
Empty mass [kg] & $m_{p, 3}$ & 2635 & 2635 & 2646 & 2952 \\
Fuel mass [kg] & $I_{s p, 3}$ & 300 & 300 & 255 & 300 \\
Specific impulse [s] & $\Delta t_{3}$ & 274 & 274 & 48 & 308 \\
Burn time [s] & $\mu_{3}$ & 5.7 & 5.7 & 5.8 & 5.7 \\
\hline Fuel-structure ratio & & & & &
\end{tabular}

- The first, called U2a in Table 2, is a missile model by Wright and Postol (2009). Based on photographs of the Unha-2 missile and data from existing missiles, they concluded that its first stage used a cluster of four Nodong motors and that the second and third stages were based on the Soviet SS-N-6 medium range ballistic missile. The SS-N-6 has a main engine flanked by two smaller Vernier thrusters 
used for attitude control. In this model, the second stage of the Unha2 uses the main engine and the thrusters of the SS-N-6, while the third stage uses only a set of thrusters.

- For missile model U2b, Wright and Postol's data are used for the first and third stages, but the second stage is different. Schiller and Schmucker (2012b) conclude, based on analysing photographs, that the second stage of the Unha-2 is based on technology of the Scud/Nodong and carries $6.2 \mathrm{~m}^{2}$ of oxidizer and $3.6 \mathrm{~m}^{2}$ of fuel. Using the respective densities of the fuel and oxidizer and by assuming that the fuel-structure ratio of his stage is the same as for the Nodong, the total mass for the second stage can be estimated. The duration of the boost-phase is found by assuming that the engine is the same as that of the Nodong, with an identical mass flow rate.

- Missile model U2c combines Postol and Wright's data for the first stage (from model U2a), the modified Nodong-based second stage (from model U2b) and a Nodong-based third stage. Data for this latter stage follows from Postol (2009).

The payload for all versions is set to $300 \mathrm{~kg}$. This includes the mass of the satellite, the aerodynamic shroud that protects the satellite during the launch and the mechanism to detach the satellite from the third stage (Wright \& Postol, 2009).

In the simulations, missile model U2c, with Nodong-based second and third stages, cannot achieve an altitude of $500 \mathrm{~km}$ at the end of its boost-phase with a pitch-angle of 0 degrees. Missile model $U 2 a$ and $U 2 b$ are sufficiently powerful to reach a circular orbit at a higher altitude than $500 \mathrm{~km}$; missile model U2b achieves a circular orbit at an altitude of $532 \mathrm{~km}$, whilst the missile based on the SS-N-6, U2a, reaches an orbit at an altitude of $638 \mathrm{~km}$. These results are shown in Fig. 5 (a). For both missiles, however, the first and second stages fall far short of the announced hazard zones. The results for simulations in which the end of the boost-phase is set to $500 \mathrm{~km}$, in Fig. 5 (b), show that both missiles can also achieve this, although the resulting orbits are now elliptical. The first stages for both missiles fall closer to the announced hazard zone, although still short. The second stages fall short of the hazard zone, but much closer to it, and U2a, with an SS-N-6 based second 
stage, falls closest. From these results it would appear that Wright and Postol's original model is the most accurate.

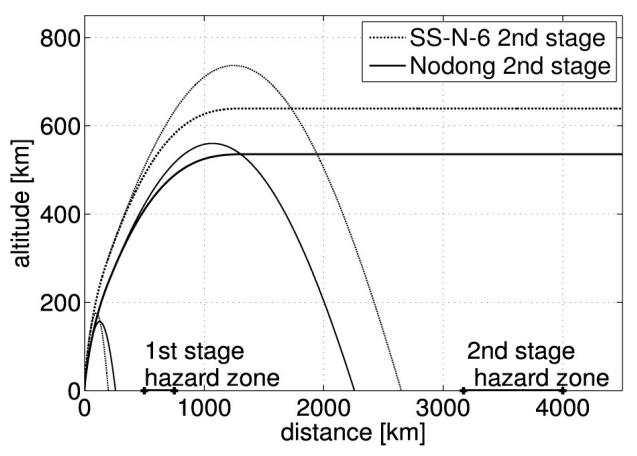

(a)

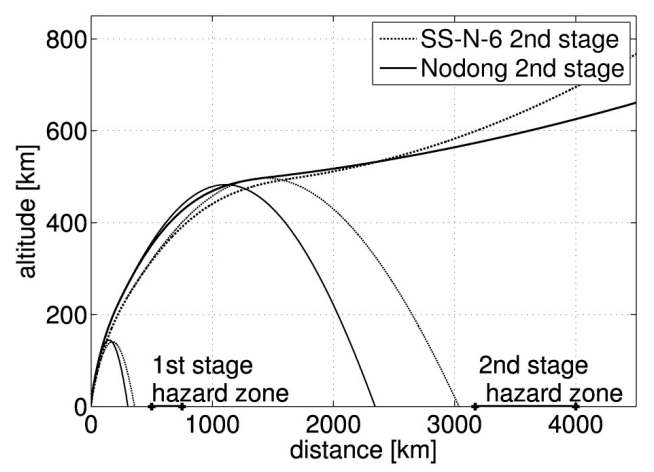

(b)

Figure 5: Simulation results for the Unha-2 with second stages based on the SS-N-6 (missile model U2a, dotted lines) and the Nodong (missile model U2b, solid lines). (a) Both missiles can achieve circular orbits, but at altitudes higher than $500 \mathrm{~km}$. (b) If the boost-phase forcibly ends at an altitude of 500 $\mathrm{km}$, elliptical orbits are reached. In neither of these cases do the $1^{\text {st }}$ and $2^{\text {nd }}$ stages impact in the hazard zones.

The fourth missile listed in Table 2 is an Unha-3, with second and third stages based on the SS-N-6. The Unha-3 has a longer third stage, see Fig. 1. The parameters for the third stage of the missile, listed as model U3 in Table 2 , are estimated by multiplying with the ratio of the respective lengths of the third stages, so $2.75 \mathrm{~m}$ vs. $2.45 \mathrm{~m}$. When launched to the south, as in the 2012 satellite launch, this missile can achieve a circular orbit at an altitude of 626 $\mathrm{km}$ and an elliptical orbit with a boost-phase ending at $500 \mathrm{~km}$. The results are shown in Fig. 6 (a).

For both the circular and the elliptical trajectories, the second stage falls into the second hazard zone and the first stage falls slightly short. It is possible to fine-tune the missile model such that the agreement is better, with a more powerful first stage and a slightly less powerful second stage. For instance, modelling the Unha-3 with an increase in the $I_{s p}$ for the first stage from 
$220 \mathrm{~s}$ to $230 \mathrm{~s}$ and a decrease for the second and third stages from $300 \mathrm{~s}$ to $274 \mathrm{~s}$, which is the value for the SS-N-6 from Pike (2011), results in a circular orbit at $500 \mathrm{~km}$, with the first stage impacting very close to the first hazard zone and a second stage impacting in the second hazard zone. This result is shown in Fig. 6 (b).

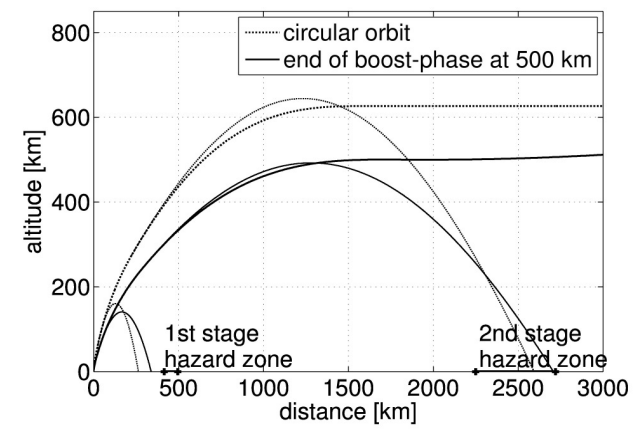

(a)

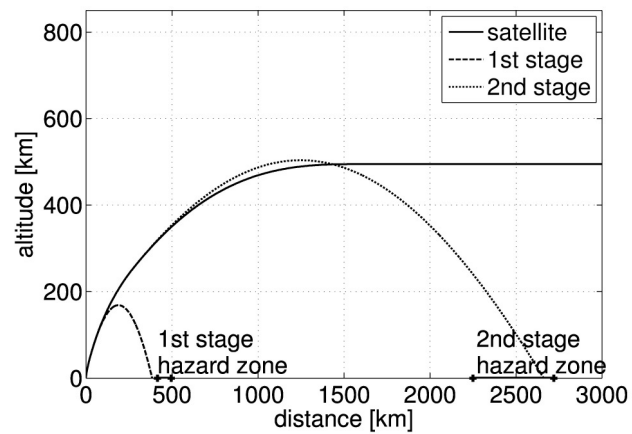

(b)

Figure 6: Direct injection to orbit using the Unha-3. (a) The Unha-3 in Table 2 , missile model U3, with a second stage based on the SS-N-6 and a lengthened third stage, reaches a circular orbit at an altitude that is too high or an elliptical orbit at $\boldsymbol{h}_{\text {orbit }}=\mathbf{5 0 0} \mathrm{km}$. (b) The Unha-3, with a larger $\boldsymbol{I}_{\boldsymbol{s} \boldsymbol{p}}$ for the first stage and smaller $\boldsymbol{I}_{\boldsymbol{s} \boldsymbol{p}}$ for the second and third stages reaches a circular orbit at the desired altitude of $\mathbf{5 0 0} \mathbf{~ k m}$.

In December 2012, the Kwangmyŏngsŏng 3-2 satellite was placed in an elliptical orbit between 500 and $584 \mathrm{~km}$. Wreckage of the first stage, including the complete oxidizer tank and parts of the engines, was recovered by the South Korean navy, $430 \mathrm{~km}$ south of the launch site, just inside the hazard zone (Blau, 2012). The wreckage confirms that the first stage indeed uses four engines and the size of the tank is similar to the model by Wright and Postol (2009). Regardless of the exact specifications, the results suggest that the Unha- 2 and 3 have a higher performance than a missile based solely on the technology of the Nodong. The third stage of the Unha-2 appears to be based on the SS-N-6, or at least on missile technology with a similar performance and, likely, the second stage as well. 


\section{North Korea's medium range ballistic missiles}

That the Unha-2 requires a more powerful engine or has a lighter structure than a missile based on the technology of the Nodong has direct implications for the theoretical performance for the Musudan and KN-08.

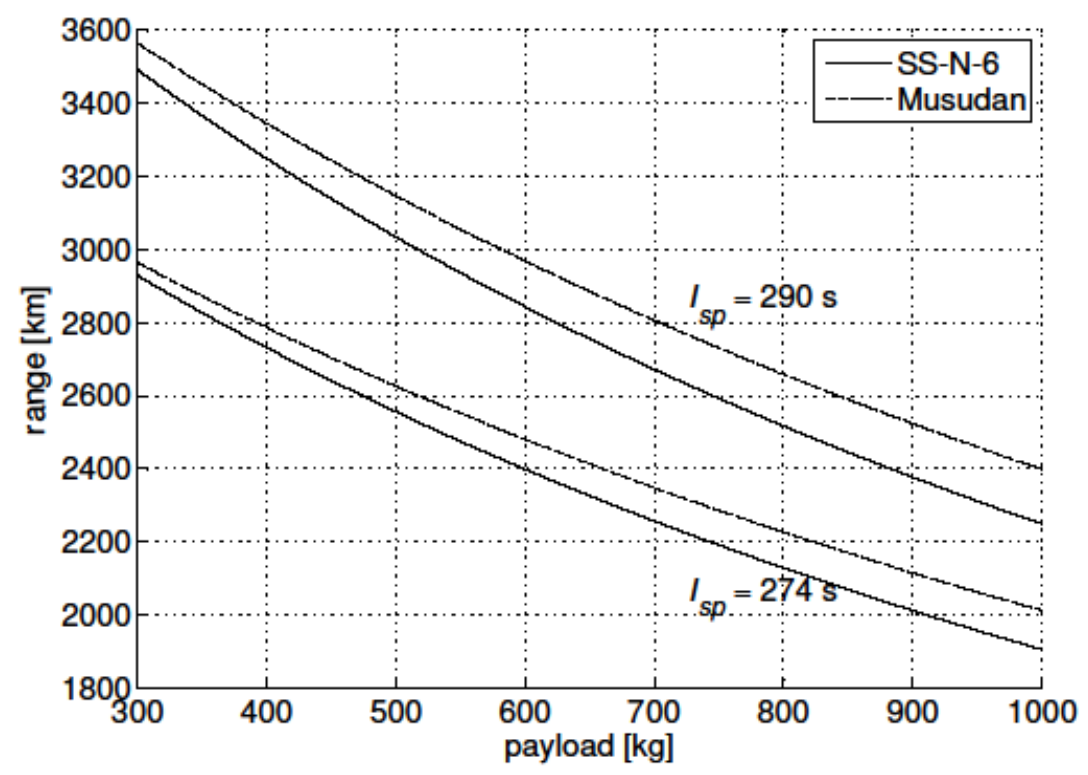

Figure 7: Ranges for the SS-N-6 (solid lines) and a Musudan missile (dashed lines) modelled as a lengthened SS-N-6 as a function of the payload. The gain in range over the SS-N-6, for the same payload, is less than $200 \mathrm{~km}$.

The results, in Fig. 7, show that the performance gain achieved by lengthening the SS-N-6 is only a few hundred kilometres, unless payload is sacrificed to accommodate the mass of the extra fuel, as was done to increase the range of the Scud-C. The calculated ranges are consistent with the results of similar calculations by Wright (2010). Even for the highest $I_{s p}$, with a $650 \mathrm{~kg}$ payload the maximum range is still considerably less than the $4000 \mathrm{~km}$ range quoted by South Korean news sources (Pollack, 2010).

The Musudan resembles the SS-N-6, but the structure of the $\mathrm{KN}-08$ is a mystery. The TEL that carried the missile during the parade in Pyongyang 
has been identified as being of Chinese origin. Its length is known and provides an essential clue to the length of the missile. Nonetheless, different analysts come up with different dimensions for the $\mathrm{KN}-08$ (Richardson, 2012a). Hansen, whose data was used for drawing Fig. 1, estimates that the diameter of the KN-08 is the same as that of the SS-N-6 and assumes that the third stage is powered by the Vernier thrusters of the SS-N-6 (Hansen, 2012). Other analysts find larger dimensions (Schiller \& Schmucker, 2012b). By appropriately scaling the SS-N-6 data, as listed in Table 1, using the lengths of the first two stages and by using Postol's data for a third stage based on the SS-N-6, the total mass of the $\mathrm{KN}-08$ is approximately 27.4 tons, excluding the payload. The launch weight therefore is $270 \mathrm{kN}$. The SS-N-6 engines deliver a thrust of $260 \mathrm{kN}$ with $I_{s p}=274 \mathrm{~s}$ and a thrust of $275 \mathrm{kN}$ for $I_{s p}=290 \mathrm{~s}$. Even the higher thrust is barely sufficient to lift the missile. The only known rocket engine available to North Korea that delivers a higher thrust is the cluster of four Nodong engines used for the first stage of the Unha-2 and 3. The diameter of the $\mathrm{KN}-08$ seems too small to be able to accommodate this, however. For the missile to be able to fly, it would need a new, as yet unknown engine. Without knowledge of this engine, it is not possible to derive accurate parameters for simulating the missile's trajectory and calculating its maximum range.

\section{Discussion}

Several assumptions are made in the model used here, as in the model by Forden (2007). The effect of wind is not taken into account, the Earth is modelled as a perfect sphere, the drag coefficient as a function of the Machnumber is the same for each stage and the thrust for each stage is assumed to be constant. The computer model could be further refined, but the calculated maximum ranges are already within a few per cent of the published ranges.

Wright and Postol (2009) have calculated trajectories for the Unha-2 with an SS-N-6 based second stage (model U2a in Table 2) in which it reaches a circular orbit at an altitude of $500 \mathrm{~km}$. This is lower than the altitude in a similar simulation here, in Fig. 5 (a), which suggests that the performance in the calculations here is overestimated. However, they have not published details of their computer model, which makes a direct comparison difficult. The Unha-3-2 actually did reach a higher and slightly elliptical orbit, which is 
consistent with the results presented in Fig. 6 (a). The pitch program used for the space launches, using a polynomial function for the angle $\varphi$ as a function of time (Eq. 5), results in the missiles reaching orbit, with discarded stages ending up close to the appropriate hazard zones, but the calculated trajectories may differ in detail from the actual trajectories. An analysis of a satellite photograph of the contrail left by the Unha-2's launch suggests that this missile followed a shallower trajectory during the first part of its flight than in the simulations presented here (Forden, 2009a). It also suggests that the actual pitch program limited the angle of attack whenever a stage was due to separate and when the missile passed through the sound barrier (Forden, 2009 b), but few details were published. Constraints such as these are not included in the model presented here. Researchers from Stanford University, who have done a similar analysis using trajectory data derived from graphs shown in North Korean television footage of the Unha-3 launch, reached the conclusion that parameters of the Unha-3 missile needed to be modified relative to Postol's model and they also had to fine-tune their pitch program (38 North, 2012). However, they too have not yet published details of the analysis.

The most important factor in the accuracy of the results is likely the limited accuracy of the missile parameters. This can be seen in Fig. 7: an increase in the specific impulse of approximately $6 \%$ leads to an increase of almost $20 \%$ in the maximum range. Calculating how the specific impulse changes with altitude would require detailed information about the engine and the nozzle geometry. The accuracy of the parameters that are given in literature and that are the basis for the parameters in Tables 1 and 2 is not always clear. For example, Postol's model of the second stage of the Unha-2, based on the SSN-6, uses a specific impulse of $300 \mathrm{~s}$ (Postol 2009), whilst according to Pike, the SS-N-6 has a specific impulse of 274-290 s (Pike 2011). Scaling the masses of the missile stages with their lengths introduces a further error. Since the length of the rocket engine does not change when the length of a stage is changed, changing the length will probably affect the fuel quantity most. This means that the amount of fuel in a shortened stage is probably overestimated, while the amount of fuel in a lengthened stage is underestimated. This error can be reduced by using missiles that are very similar as the basis of the estimated parameters, but better estimates require more detailed knowledge of the interior configuration of the missile. 
Table 3: Effect of changing booster parameters on the maximum range of the SS-N-6 (relative to the parameters listed in Table 1) and parameters of the second stage of the Unha-2 (missile model U2a in Table 2) on the altitude of the orbit reached.

\begin{tabular}{|c|c|c|c|c|}
\hline Change in parameter [\%] & -10 & +10 & -10 & +10 \\
\hline Parameter changed & \multicolumn{2}{|c|}{$\begin{array}{l}\text { SS-N-6 } \\
\text { change in range [\%] }\end{array}$} & \multicolumn{2}{|c|}{$\begin{array}{l}\text { Unha-2 } \\
\text { change in } h_{\text {orbit }} \text { [\%] }\end{array}$} \\
\hline Payload mass & +4 & -5 & +3 & -3 \\
\hline Stage mass & -5 & +4 & -0.4 & +0.1 \\
\hline Burn time & +2 & -2 & -1 & +0.8 \\
\hline Fuel-structure ratio & -9 & +9 & -2 & +2 \\
\hline$I_{s p}$ & -27 & +27 & -10 & +10 \\
\hline
\end{tabular}

To make the influence of the uncertainty in the missile parameters clearer, simulations were done in which parameters were increased and decreased by $10 \%$, one at a time. The effect on the range was studied for the (single-stage) SS-N-6, with results listed in Table 3. Errors of $\pm 10 \%$ in either the stage mass or the payload mass change the range by at most $5 \%$. The effect of changing the burn time is smaller, which is to be expected. Decreasing it increases the acceleration, but simultaneously decreases the time during which the missile accelerates and, consequently, the gain in the overall velocity and the range is small. Changing the fuel-structure ratio has a larger effect, since increasing it leads to a larger acceleration that lasts equally long. The specific impulse has the largest impact, as was already suggested by Fig 7. Table 3 also includes results of a second set of simulations that show the effect of errors in the parameters on the orbit that can be reached by the Unha-2. Since one of the central questions is whether the second stage of this missile is based on the technology of the SS-N-6 or of the Nodong, only parameters of the second stage were changed, using missile model U2a as the starting point. Here too, the effect of changing the parameters is relatively small, except for the effect of the specific impulse. Because of this, a final simulation was done using missile model U2c, with second and third stages based on the Nodong, in which the specific impulse of all three stages was increased by $10 \%$. The missile does reach orbit, but at an altitude of only 409 $\mathrm{km}$, which is still considerably lower than the intended $500 \mathrm{~km}$. 


\section{Conclusion}

Regardless of the exact values of the parameters and of whether the model used here overestimates the performance, an Unha-2 that is solely based on the Scud/Nodong cannot reach the correct orbit. Based on the (intended) satellite trajectories and the announced impact zones, the second stage of the Unha-2 and the Unha-3/3-2 indeed seems to be more advanced than Scudderivatives. This represents a technological leap forward for North Korea. The Musudan missile, when modelled as a lengthened SS-N-6, offers a slightly larger range than the SS-N-6 and, as such, has the potential to be a much bigger threat to the region than the Nodong. However, despite the successful launch of the Unha-3-2, the failure of the launches of the Taepodong-2, the Unha-2 and Unha-3 and the apparent lack of flight tests of the Musudan missile itself, indicate that it is far from an operation system. The rocket engine used in the second stage of the Unha-2 and Unha-3 does not deliver enough thrust to power the KN-08. For this missile to be anything other than an elaborate propaganda tool, it would need a new higher thrust engine. Developing it into an operational system may exceed North-Korea's ability and, in any case, would require an extensive test program.

\section{References}

38 North (2012, April 10). GoogleEarth 3D Model of the Unha-3 Flight Path. Retrieved April 14, 2012, from 38 North: http://38north.org/2012/04/tongchang041012/

Anderson, J. D., Jr. (2008). Introduction to Flight (6th ed.). New York: McGraw-Hill International Edition.

BBC (2012, December 13). UN Security Council condemns North Korea rocket launch. Retrieved December 13, 2012, from BBC News Asia: http://www.bbc.co.uk/news/worldasia-20697922

Blau, P. (2012, December 15). South Korea retrieves North Korean Unha-3 Rocket debris. Retrieved January 8, 2013, from spaceflight101: http://www.spaceflight101.com/unha-3launch-updates-kwangmyongsong-3-2.html

CGWIC (2011). User's manual. Retrieved November 20, 2012, from China Great Wall Industry Corporation: http://www.cgwic.com/LaunchServices/Download/manual/index.html

Christy, R. (2012a, April). Unha 3/ Kwangmyongsong 3 Launch failure. Retrieved May 10, 2012, from Zarya: http://www.zarya.info/Diaries/NKorea/Unha3Impact.php 
Christy, R. (2012b, December). Kwamgmyongsong 3-2 Launch. Retrieved December 3, 2012, from Zarya:

http://www.zarya.info/Diaries/NKorea/Kwangmyongsong32NOTAM.php

Covault, C. (2009, April 10). North Korean rocket flew further than earlier thought. Retrieved October 19, 2012, from Spaceflight Now:

http://spaceflightnow.com/news/n0904/10northkorea/

Fetter, S. (1990). A ballistic missile primer. Retrieved November 21, 2012, from Publications of Steve Fetter: http://faculty.publicpolicy.umd.edu/sites/default/files/fetter/files/1990MissilePrimer.pdf

Forden, G. E. (2007). GUI Missile Flyout: A General Program for Simulating Ballistic Missiles. Science and Global Security , 15, 133-146.

Forden, G. E. (2009a, April 20). DPRK: U'nha-2 powered trajectory. Retrieved November 6, 2012, from Arms Control Wonk: http://forden.armscontrolwonk.com/archive/2262/dprkunha-2-powered-trajectory

Forden, G. E. (2009b, May 15). DPRK: Why did the 2006 launch fail? Retrieved November 6, 2012, from Arms Control Wonk:

http://forden.armscontrolwonk.com/archive/2298/dprk-why-did-the-2006-launch-fail

Hansen, N. (2012, May 4). North Korea's new long-range missile: fact or fiction? Retrieved October 16, 2012, from 38 North: http://38north.org/2012/05/nhansen050412/

Hecker, S. S., \& Carlin, R. (2012). North Korea in 2011: Countdown to Kim-il-Sungs centenary. Bulletin of the atomic scientists , 68 (1), 50-60.

Global Security (2009, March 12). Information on the launch of an experimental communications satellite Kwangmyŏngsŏng-2. Retrieved October 17, 2012, from Globalsecurity.org: http://www.globalsecurity.org/space/library/news/2009/space090312-imo01.htm

KNCA (2012, March 16). DPRK to Launch Application Satellite. Retrieved April 16, 2012 , from Korean News Service in Tokyo: http://www.kcna.co.jp/item/2012/201203/news16/20120316-20ee.html

Lennox, D. (Ed.). (2009). Jane's Strategic Weapons Systems (Vol. 50). Surrey: Jane's Information Group.

Lewis, J. (2012a, April 19). DPRK ICBM Items. Retrieved April 27, 2012, from Arms Control Wonk: http://lewis.armscontrolwonk.com/archive/5150/dprk-icbm-items

Lewis, J. (2012b, June). Origins of the Musudan IRBM. Retrieved July 3, 2012, from Arms Control Wonk: http://lewis.armscontrolwonk.com/archive/5337/origins-of-the-musudanirbm

NASA (1968, December). Saturn V Press Kit. Retrieved November 26, 2012, from MSFC History Office: http://history.msfc.nasa.gov/saturn_apollo/saturnv_press_kit.html

Pellerin, C. (2012, April 12). Northcom Acknowledges North Korean Missile Launch Failure. Retrieved May 10, 2012, from American Forces Press: http://www.defense.gov/news/newsarticle.aspx?id=67920

Pike, J. (2011, July 24). R-27 / SS-N-6 SERB \& SS-NX-13. Retrieved April 24, 2012, from GlobalSecurity.org: http://www.globalsecurity.org/wmd/world/russia/r-27-specs.htm 
Pinkerton, D. A. (2008). The North Korean ballistic missile program. Carlisle, PA.: Strategic Studies Institute, US Army War College.

Pollack, J. (2010, October 10). North Korea Debuts an IRBM. Retrieved April 24, 2012, from Arms Control Wonk: http://pollack.armscontrolwonk.com/archive/3351/NorthKorea-debuts-an-irbm

Postol, T. (2009). A Technical Assessment of Iran's Ballistic Missile Program, Technical Addendum to the Joint Threat Assessment on Iran's Nuclear and Missile Potential. EastWest Institute.

Richardson, D. (2012a, June). North Korea flexes its missile muscle. Jane's Missiles and Rockets, 16 (6), pp. 1-2.

Richardson, D. (2012b, July). Analysts struggle to understand North Korea's KN-08. Jane's Missiles and Rockets 16 (7), pp. 1-2.

Schiller, M. (2012). Characterizing the North Korean Nuclear Missile Threat. Santa Monica: RAND Corporation.

Schiller, M., \& Schmucker, R. H. (2012a). Explaining the Musudan. Munich: Schmucker Technologie.

Schiller, M., \& Schmucker, R. H. (2012b). The Assumed KN-08 Technology. Munich: Schmucker Technologie.

Sessions, J. (2008). Ballistic Missile Defense: A National Priority. Strategic Studies Quarterly, 22-30.

Shampine, L.F., Reichelt, M. W. (1997), The Matlab ODE Suite. SIAM Journal on Scientific Computing 18 (1), 1-22.

Vallado, D.A. (2007) Fundamentals of Astrodynamics and applications (3rd ed.), New York : Springer.

Vick, C. P. (2012, October 19). Nodong. Retrieved October 19, 2012, from Federation of American Scientists: http://www.fas.org/nuke/guide/dprk/missile/nd-1.htm

Williams, M. (2012, March 21). Exclusive: North Korea's expected rocket trajectory. Retrieved April 26, 2012, from North Korea Tech:

http://www.northkoreatech.org/2012/03/21/exclusive-north-koreas-expected-rockettrajectory/

Wright, D. (2010, October 12). More on Musudan range estimates. Retrieved November 5, 2012, from All Things Nuclear: http://allthingsnuclear.org/more-on-musudan-rangeestimates/

Wright, D. (2012, April 8). A comparison of North Korea's Unha-2 and Unha-3. Retrieved October 19, 2012, from All things nuclear: http://allthingsnuclear.org/a-comparison-ofnorth-koreas-unha-2-and-unha-3/

Wright, D., \& Postol, A. T. (2009, June 29). A post-launch examination of the Unha-2. Retrieved April 24, 2012, from The bulletin of the atomic scientist web edition: http://thebulletin.org/web-edition/features/post-launch-examination-of-the-unha-2

Zarchan, P., (1997). Tactical and Strategic Missile Guidance (3rd ed.), Reston, Virginia: AIAA 